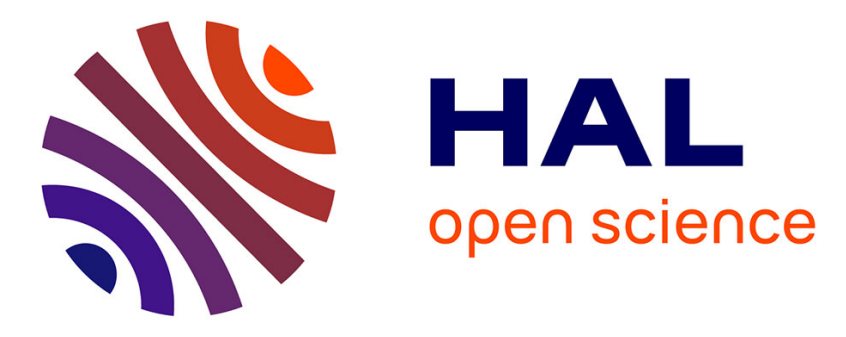

\title{
ECG Fiducial Points Extraction by Extended Kalman Filtering
}

\author{
Mahsa Akhbari, Mohammad B. Shamsollahi, Christian Jutten
}

\section{To cite this version:}

Mahsa Akhbari, Mohammad B. Shamsollahi, Christian Jutten. ECG Fiducial Points Extraction by Extended Kalman Filtering. TSP 2013 - 36th International Conference on Telecommunications and Signal Processing, Jul 2013, Rome, Italy. pp.628-632. hal-00853768

\section{HAL Id: hal-00853768 https://hal.science/hal-00853768}

Submitted on 27 Sep 2013

HAL is a multi-disciplinary open access archive for the deposit and dissemination of scientific research documents, whether they are published or not. The documents may come from teaching and research institutions in France or abroad, or from public or private research centers.
L'archive ouverte pluridisciplinaire HAL, est destinée au dépôt et à la diffusion de documents scientifiques de niveau recherche, publiés ou non, émanant des établissements d'enseignement et de recherche français ou étrangers, des laboratoires publics ou privés. 


\title{
ECG Fiducial Points Extraction by Extended Kalman Filtering
}

\author{
Mahsa Akhbari, Mohammad B. Shamsollahi and Christian Jutten
}

\begin{abstract}
Most of the clinically useful information in Electrocardiogram (ECG) signal can be obtained from the intervals, amplitudes and wave shapes (morphologies). The automatic detection of ECG waves is important to cardiac disease diagnosis. In this paper, we propose an efficient method for extraction of characteristic points of ECG. The method is based on a nonlinear dynamic model, previously introduced for generation of synthetic ECG signals. For estimating the parameters of model, we use an Extendend Kalman Filter (EKF). By introducing a simple AR model for each of the dynamic parameters of Gaussian functions in model and considering separate states for ECG waves, the new EKF structure was constructed. Quantitative and qualitative evaluations of the proposed method have been done on Physionet QT database (QTDB). This method is also compared with a method based on Partially Collapsed Gibbs Sampler (PCGS). Results show that the proposed method can detect fiducial points of ECG precisely and mean of estimation error of all FPs (except Ton) do not exceed five samples $(20 \mathrm{msec})$.
\end{abstract}

Keywords-Electrocardiogram (ECG), Extended Kalman Filter (EKF), Characteristic Waves, Fiducial Points Extraction, Segmentation.

\section{INTRODUCTION}

Electrocardiogram (ECG) is a non-invasive, safe and quick method for cardiac disease diagnosis. Fiducial Points (FPs) in an ECG signal are the location of peak, onset and offset of waveforms which have clinically useful information for physicians. "Fiducial Point Extraction" and "Segmentation" of ECG can be a first step in many ECG analysis tasks to determine as accurately as possible the peak, onset and offset locations of the ECG waves.

Up to now, different methods have been used for detecting the QRS complex. Some of them were discussed in [1]. These methods are based on mathematical functions, filtering approaches (digital filters, adaptive filters), different mathematical transformations (Wavelet, Hilbert) and classification methods (neural network approaches, Support Vector Machine (SVM), fuzzy C-means algorithm) [1]. Some methods have also been used for $\mathrm{P}$ and $\mathrm{T}$ wave delineation and estimation such as Partially Collapsed Gibbs Sampler (PCGS) [2], [3], Hidden Markov Models (HMM) [4] and mathematical morphology methods [5].

This work partially supported by scholarship of French Embassy. The authors would like to thank Mr. Ali Almasi and Ms. Nasim Montazeri, for their helps.

M. Akhbari is with the BiSIPL, Department of Electrical Engineering, Sharif university of Technology, Tehran, Iran. She is also now with the GIPSALab, Grenoble, France. (Corresponding author, email: mahsa.akhbari@gipsalab.grenoble-inp.fr

M. B. Shamsollahi is with the BiSIPL, Department of Electrical Engineering, Sharif University of Technology, Tehran, Iran. (email: mbshams@sharif.edu)

C. Jutten is with the GIPSA-Lab, Grenoble, and Institut Universitaire de France, France. (email: christian.jutten@gipsa-lab.grenoble-inp.fr)
A nonlinear dynamical model for generation of synthetic ECG signals has been recently developed by McSharry et al. [6]. Sameni et al. [7] transformed this model and proposed an Extended Kalman Filter (EKF) algorithm for denoising ECG signals ("EKF2"). Sayadi et al. modified the EKF2 framework and added parameters of ECG dynamical model as states to EKF2 and introduced the "EKF17" approach [8]. They also described a Gaussian wave-based state space model whose each characteristic wave of ECG has been considered as a state ("EKF4") [9].

In this paper, we propose a method for detection the fiducial points of ECG signal. In our method by taking the idea of EKF4 and EKF17 approaches, we introduce a simple AR model for parameters of Gaussian functions in ECG dynamical model and also consider three separate states for ECG waves. In brief, we consider 25 parameters of ECG signal as states of an EKF and we will find peak, onset and offset of all characteristic waves (QRS complex, $\mathrm{P}$ and $\mathrm{T}$ waves) of ECG signal. For validation of our method, we will use QT database (QTDB) [10], [11] which has ECG signals with cardiologist annotations. We also compare our method with a method based on Partially Collapsed Gibbs Sampler (PCGS) [2], [3].

In our previous work [12], we have proposed a method (which we called it "EKF25old") for finding the fiducial points of ECG signals. In this paper we extend our method and modify it in order to find fiducial points more exactly.

Due to space limitations, basics of EKF are not discussed in this paper. Details of them can be found in [13], [7], [14]. In this paper we will benefit of previous EKF approaches ("EKF2", "EKF17" and "EKF4"). These approaches are discussed in Section II and our previous approach ("EKF25old) is discussed in section III. In Section IV, we explain our proposed method ("EKF25new") for fiducial points extraction. In section $\mathrm{V}$, we present the results of applying the proposed method for ECG signals of QT database. Finally, discussion and conclusions are provided in Section VI.

\section{Previous EKF Approaches}

McSharry et al. [6] have proposed a synthetic ECG generator, which is based on a nonlinear dynamic model. Details of this model can be found in [6]. Sameni et al. [7] transformed these dynamic equations into the polar form to obtain a simpler compact set, with the simplified discrete form shown as:

$$
\left\{\begin{array}{l}
\varphi_{k+1}=\left(\varphi_{k}+\omega \delta\right) \bmod (2 \pi) \\
z_{k+1}=-\sum_{i} \delta \frac{\alpha_{i} \omega}{b_{i}^{2}} \Delta \theta_{i} \exp \left(-\frac{\Delta \theta_{i}^{2}}{2 b_{i}^{2}}\right)+z_{k}+\eta
\end{array}\right.
$$

where $\Delta \theta_{i}=\left(\varphi_{k}-\theta_{i}\right) \bmod (2 \pi), \delta$ is the sampling time, $\eta$ is a random additive noise that models the inaccuracies of the 
dynamic model and the summation over $i$ is taken over the number of Gaussian functions used for modeling the shape of the ECG. The $\alpha_{i}, b_{i}$ and $\theta_{i}$ terms in (1) correspond to the amplitude, angular spread and location of the Gaussian functions and $\omega$ is the angular velocity represents the $R R$ interval variability.

Sayadi et al. extended EKF2 framework and added parameters of 5 Gaussian functions in (1) as states to EKF2. In fact in this approach, 2 states were the same as in EKF2 and 15 other states were added, so that the method was called "EKF2+15" or briefly "EKF17". This approach was used for ECG denoising, compression and beat segmentation [8].

Sayadi et al. also described a Gaussian wave-based state space model [9] whose each characteristic wave, i.e. P, QRS and $\mathrm{T}$ has been considered as a state. As this structure had 4 states, they called it "EKF4" and used it for ECG arrhythmia detection especially PVC detection. In this model they considered two Gaussian functions for $\mathrm{P}$ and $\mathrm{T}$ waves.

\section{OUR Previous Method ("EKF25OLD” APPROACH)}

In our previous work [12], we have modified the previous EKF approaches. Discrete state and observation equations of our previous model are defined in (2) and (3), respectively.

$$
\left\{\begin{array}{l}
\varphi_{k+1}=\left(\varphi_{k}+\omega \delta\right) \bmod (2 \pi) \\
P_{k+1}=-\sum_{i \in\left\{P_{1}, P_{2}\right\}} \delta \frac{\alpha_{i k} \omega}{b_{i k}^{2}} \Delta \theta_{i k} \exp \left(-\frac{\Delta \theta_{i k}^{2}}{2 b_{i k}^{2}}\right)+P_{k}+\eta_{P} \\
C_{k+1}=-\sum_{i \in\{Q, R, S\}} \delta \frac{\alpha_{i k} \omega}{b_{i k}^{2}} \Delta \theta_{i k} \exp \left(-\frac{\Delta \theta_{i k}^{2}}{2 b_{i k}^{2}}\right)+C_{k}+\eta_{C} \\
T_{k+1}=-\sum_{i \in\left\{T_{1}, T_{2}\right\}} \delta \frac{\alpha_{i k} \omega}{b_{i k}^{2}} \Delta \theta_{i k} \exp \left(-\frac{\Delta \theta_{i k}^{2}}{2 b_{i k}^{2}}\right)+T_{k}+\eta_{T} \\
\alpha_{i, k+1}=\alpha_{i, k}+u_{j, k}, j=\{1, \cdots, 7\} \\
b_{i, k+1}=b_{i, k}+u_{j, k}, j=\{8, \cdots, 14\} \\
\theta_{i, k+1}=\theta_{i, k}+u_{j, k}, j=\{15, \cdots, 21\} \\
i \in\left\{P_{1}, P_{2}, Q, R, S, T_{1}, T_{2}\right\} \\
\Phi_{k}=\varphi_{k}+v_{1 k} \\
s_{k}=P_{k}+C_{k}+T_{k}+v_{2 k}
\end{array}\right.
$$

In this model, the first state is the phase of the ECG. The second, third and forth ones are the different waves of ECG which are separately considered as a state. The parameters of the Gaussian functions are considered as hidden-state variables (states 5 to 21) with first order AR dynamics but without corresponding observations. As this model has 25 states and as it was our previous model, we call it "EKF25old". In this model, we assumed that the ECG observation can be defined as a summation of $P, C$ and $T$ states. This assumption was not wrong but it had drawback in estimating the ECG waves which we will discuss this matter in section IV. Fig. 1 shows the estimated waves and ECG signal by "EKF25old" approach for a signal of QT database ("Sel16786"). In this figure, we can see that the waves are estimated correctly but they have a rising and falling drifts, compensated in the estimated ECG which is the sum of estimated waves. These drifts may be due to this fact that in this model, we have only one observation (based on 3 states) which corresponds to original ECG.

In [12], we have used a blockdiagram (Fig. 2) for finding the peak, onset and offset of normal ECG signals. In this
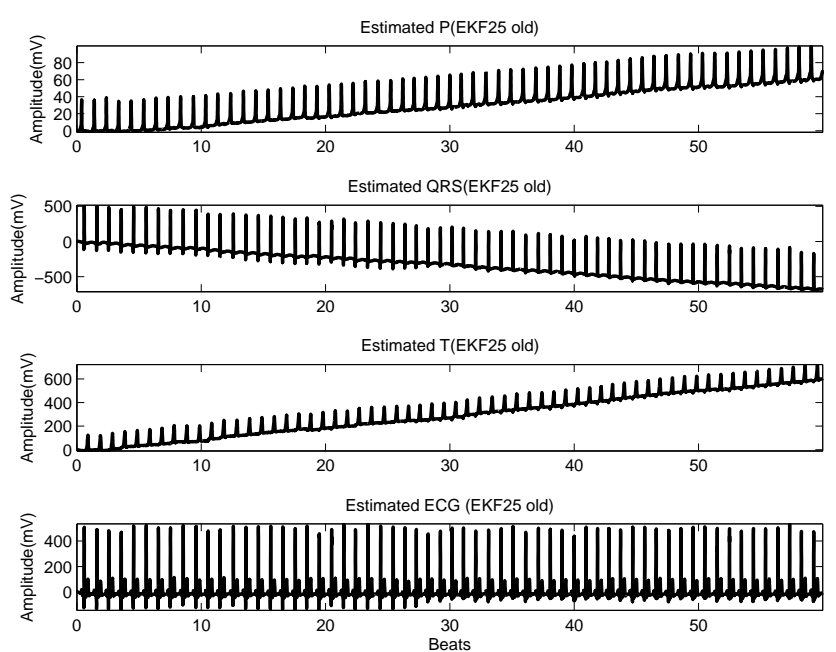

Fig. 1. Estimated Waves and ECG by EKF25old approach.

blockdiagram, at first all states of the model are estimated by "EKF25old" approach. Method which was used for finding the peak of waves, consists of three steps:

- Using "peak detection" method (finding the Maxima) for estimated waves $(\hat{P}, \hat{C}$ and $\hat{T})$ and finding their peaks which are called $P_{P}, P_{C}$ and $P_{T}$.

- $\theta_{i} \mathrm{~s}$ (peaks of Gaussian functions) are 7 states which are estimated by "EKF25old".

- Using a decision rule like (4) to find the final peak points of ECG $\left(\Theta_{P}, \Theta_{R}\right.$ and $\left.\Theta_{T}\right)$, which $s_{k}$ is the observed (original) ECG signal.

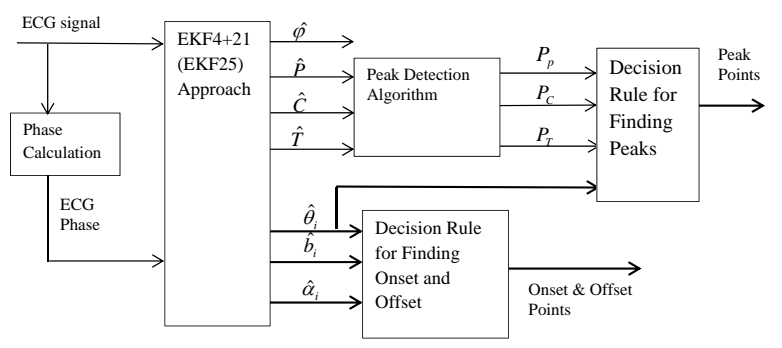

Fig. 2. Blockdiagram for the proposed "EKF25old" Approach.

$$
\begin{aligned}
& \Theta_{P}=\operatorname{argmax}\left(s_{k}\left(\theta_{P 1}\right), s_{k}\left(\theta_{P 2}\right), s_{k}\left(P_{P}\right)\right) \\
& \Theta_{R}=\operatorname{argmax}\left(s_{k}\left(\theta_{R}\right), s_{k}\left(P_{C}\right)\right) \\
& \Theta_{T}=\operatorname{argmax}\left(s_{k}\left(\theta_{T 1}\right), s_{k}\left(\theta_{T 2}\right), s_{k}\left(P_{T}\right)\right)
\end{aligned}
$$

In order to find the onset and offset of the waveforms, we first use the approximately $99 \%$ confidence bound for the termination of Gaussian functions and compute $i_{\text {on }}=\theta_{i}-3 b_{i}$, $i \in\left\{P_{1}, P_{2}, Q, R, S, T_{1}, T_{2}\right\}$ to find their onset and offsets. Then we use a decision rule like (5) to find the final onset and offset of $\mathrm{P}$ and $\mathrm{T}$ waves, here $\beta_{i}, \lambda_{i}, i=\{1, \cdots, 4\}$ are real positive coefficients. Results of this approach are presented in [12].

$$
\begin{array}{ll}
P_{\text {on }}=\beta_{1} P_{1 \text { on }}+\lambda_{1} P_{2 \text { on }}, & P_{\text {off }}=\beta_{2} P_{1 \text { off }}+\lambda_{2} P_{2 \text { off }} \\
T_{\text {on }}=\beta_{3} T_{1 \text { on }}+\lambda_{3} T_{2 \text { on }}, & T_{\text {off }}=\beta_{4} T_{1 \text { off }}+\lambda_{4} T_{2 \text { off }}
\end{array}
$$




\section{Our New Proposed Method (“EKF25NEW”)}

In section III, we have explained about our previous model for finding fiducial points of ECG signal. As we discussed there, the previous model has a drawback which the estimated waves had rising and falling drifts. In that model, we had not used the estimated waves directly and we just used a peak detection method for finding their peaks which, despite the drift of estimated waves, peak of waves can be estimated accurately.

So in this paper, we first modify the observation equations of the previous model. The new observation equations are defined in (6). Here we consider four observations for our model. The first one corresponds to phase observation and others correspond to ECG observation in $\mathrm{P}, \mathrm{C}$ and $\mathrm{T}$ intervals, respectively.

$$
\begin{aligned}
& \Phi_{k}=\varphi_{k}+v_{1 k} \\
& P P_{k}=P_{k}+v_{2 k} \\
& C C_{k}=C_{k}+v_{3 k} \\
& T T_{k}=T_{k}+v_{4 k}
\end{aligned}
$$

In fact we determine three windows for segmenting the original ECG and finding the $P P_{k}, C C_{k}$ and $T T_{k}$ observations. Here we use windows which are the difference of two sigmoid functions and have almost soft rising and falling edges. Fig. 3 shows these windows for $\mathrm{P}, \mathrm{C}$ and $\mathrm{T}$ intervals. The begining and end of these windows are defined corresponding to the phase of ECG. In fact we assume that $\mathrm{P}, \mathrm{C}$ and $\mathrm{T}$ intervals correspond to ECG phase in interval $[-\pi,-\pi / 6],[-\pi / 6, \pi / 6]$ and $[\pi / 6, \pi]$ respectively. It is important to note that this assumption for normal ECG signals is almost valid. These windows are defined in (7) and the shape of the windows is controlled with $\gamma$, set here to $\gamma=30$. Observations $P P_{k}$, $C C_{k}$ and $T T_{k}$ in (6) are calculated by multiplying the original (observed) ECG signal and windows defined in (7).

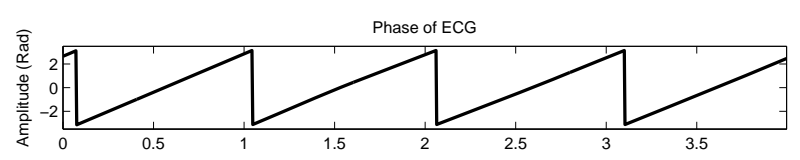

P Interval
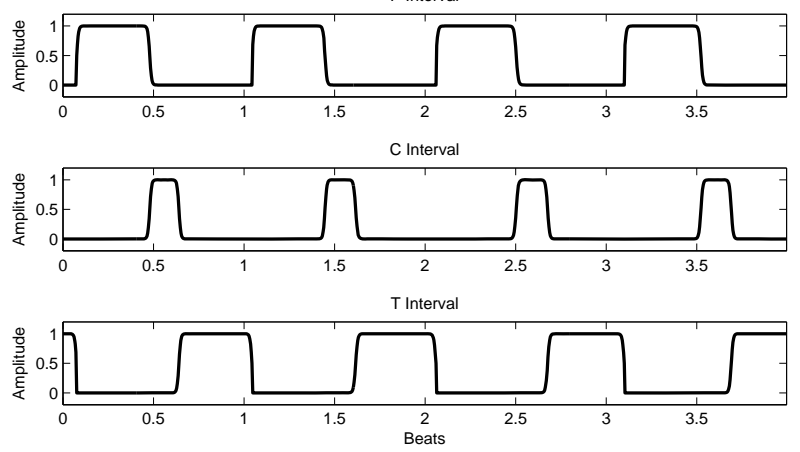

Fig. 3. ECG Phase and Windows for P, C and T Intervals.

$$
\begin{aligned}
& (P w)_{k}=\frac{1}{1+\exp ^{-\gamma\left(\Phi_{k}-(-\pi)\right)}}-\frac{1}{1+\exp ^{-\gamma\left(\Phi_{k}-(-\pi / 6)\right)}} \\
& (C w)_{k}=\frac{1}{1+\exp ^{-\gamma\left(\Phi_{k}-(-\pi / 6)\right)}}-\frac{1}{1+\exp ^{-\gamma\left(\Phi_{k}-(\pi / 6)\right)}} \\
& (T w)_{k}=\frac{1}{1+\exp ^{-\gamma\left(\Phi_{k}-(\pi / 6)\right)}}-\frac{1}{1+\exp ^{-\gamma\left(\Phi_{k}-(\pi)\right)}}
\end{aligned}
$$

So in this paper we propose a new model whose discrete states and observation equations are defined in (2) and (6), respectively. We call this model "EKF25new" and we will use it for finding fiducial points of ECG.

Another problem in our previous model ("EKF25old" approach) is that, in that model we first found the onset and offset of P1, P2, T1 and T2 waves and then we used a decision rule (equation (5)) to find the final onset and offset of $\mathrm{P}$ and $\mathrm{T}$ waves. This method is heuristic and time consuming, and requires to estimate proper $\beta_{i}$ and $\lambda_{i}$ coefficients.

Here, for finding the peak, onset and offset of waves, we use a new method which is explained below. As discussed in previous sections, we use the McSharry model which assumes that each ECG beat is a combination of N Gaussian functions (here $N=7$ ) and each Gaussian function is defined by parameters $\alpha_{i}, b_{i}$ and $\theta_{i}$ (amplitude, angular spread and location of the Gaussian functions). We first estimate the Gaussian parameters (states 5 to 21) by "EKF25new" approach, then we construct the P1, P2, Q, R, S, T1 and T2 Gaussian functions (equation (8)).

$$
i(\theta)=\hat{\alpha}_{i} \exp \left(-\frac{\left(\theta-\hat{\theta}_{i}\right)^{2}}{2 \hat{b}_{i}^{2}}\right), i \in\left\{P_{1}, P_{2}, Q, R, S, T_{1}, T_{2}\right\}
$$

For finding the peak of $\mathrm{P}, \mathrm{QRS}$ and $\mathrm{T}$ waves, we use the constructed $P 1+P 2, Q+R+S$ and $T 1+T 2$ Gaussian functions and find the maximum of these functions as a peak of waves of original ECG. Equation (9) explains this method. The advantage of the method is to use a clean signal for finding the peaks of waves. So we can find them correctly.

$$
\begin{aligned}
& \Theta_{P}=\operatorname{argmax}\left(P_{1}(\theta)+P_{2}(\theta)\right) \\
& \Theta_{R}=\operatorname{argmax}(Q(\theta)+R(\theta)+S(\theta)) \\
& \Theta_{T}=\operatorname{argmax}\left(T_{1}(\theta)+T_{2}(\theta)\right)
\end{aligned}
$$

By defining a suitable confidence bound $(\epsilon \%)$ for the termination of constructed $P 1+P 2, Q, S$ and $T 1+T 2$ Gaussian functions, we can detect the onset and offset of ECG waves. The onset and offset of $\mathrm{P}, \mathrm{QRS}$ and $\mathrm{T}$ waves are detected by equations of (10) and (11). First, we compute theoretically:

$$
\begin{aligned}
& A_{P}=\int_{-\infty}^{\infty}(P 1(\theta)+P 2(\theta)) d \theta \\
& A_{Q}=\int_{-\infty}^{\infty} Q(\theta) d \theta \\
& A_{S}=\int_{-\infty}^{\infty} S(\theta) d \theta \\
& A_{T}=\int_{-\infty}^{\infty}(T 1(\theta)+T 2(\theta)) d \theta
\end{aligned}
$$

Then we determine numerically:

$$
\begin{aligned}
& \text { Pon } \mid \int_{-\infty}^{P o n}(P 1(\theta)+P 2(\theta)) d \theta=0.01 \epsilon A_{P} \\
& \text { Poff } \mid \int_{P o f f}^{\infty}(P 1(\theta)+P 2(\theta)) d \theta=0.01 \epsilon A_{P} \\
& \text { QRSon } \mid \int_{-\infty}^{Q R S o n} Q(\theta) d \theta=0.01 \epsilon A_{Q} \\
& \text { QRSoff } \mid \int_{Q R S o f f}^{\infty} S(\theta) d \theta=0.01 \epsilon A_{S} \\
& \text { Ton } \mid \int_{-\infty}^{T o n}(T 1(\theta)+T 2(\theta))=0.01 \epsilon A_{T} \\
& \text { Toff } \mid \int_{T o f f}^{\infty}(T 1(\theta)+T 2(\theta))=0.01 \epsilon A_{T}
\end{aligned}
$$

\section{RESUlts}

For validation of our method, we use QT database which has ECG signals with cardiologist annotations. All records of database were sampled at $250 \mathrm{~Hz}$. Details can be found in [10], [11]. Fig. 4 shows the estimated $\mathrm{P}$ and $\mathrm{T}$ waves by 
"EKF25old" and "EKF25new" approaches. We can see that estimated waves by "EKF25old" have rising and falling drifts whereas those estimated by "EKF25new" have no drift.

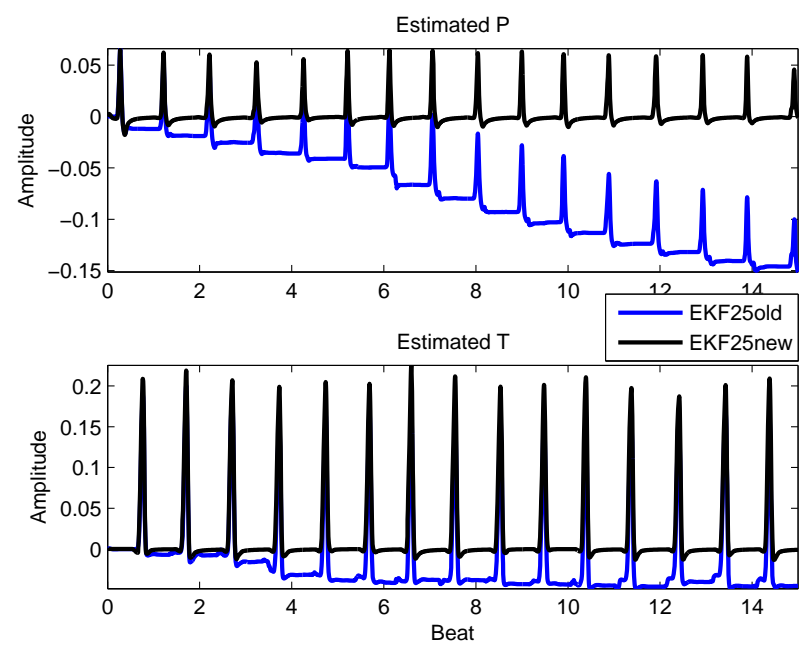

Fig. 4. Estimated $\mathrm{P}$ and $\mathrm{T}$ waves by EKF25old and EKF25new approaches.

In this paper, we find fiducial points for three normal ECG signals ("Sel17453", "Sel 16786" and "Sel16795") from QTDB. First we use a median filter for removing baseline of signals. By following the procedure which is discussed in section IV, fiducial points of ECG have been detected. As discussed there, for finding the onset and offset of waves we use estimated Gaussian functions and define a suitable confidence bound $(\epsilon \%)$ for the termination of Gaussian functions. First we consider five different values for $\epsilon$ and find the mean and standard deviation of estimation error (time differences between cardiologist annotations (considered as ground truth) and results of the proposed method). These values are reported in Tables I and II.

TABLE I

MEAN OF ERRORS (MSEC) BETWEEN ESTIMATED FPS AND MANUAL ANNOTATIONS FOR DIFFERENT VALUES OF $\epsilon$.

\begin{tabular}{lccccc}
\hline \hline FPs & $\epsilon=1 \%$ & $\epsilon=2 \%$ & $\epsilon=3 \%$ & $\epsilon=4 \%$ & $\epsilon=5 \%$ \\
\hline P on & 0.97 & 11.95 & 17.31 & 20.58 & 23.8 \\
P off & 12.73 & 3.07 & -1.17 & -3.31 & -5.56 \\
QRS on & -26.73 & -22.53 & -20.19 & -18.92 & -17.17 \\
QRS off & 32 & 19.02 & 15.65 & 13.95 & 12.53 \\
T on & 47.6 & 65.03 & 72.19 & 75.5 & 79.9 \\
T off & 0.97 & -11.9 & -17.56 & -20.87 & -24.29 \\
\hline \hline
\end{tabular}

TABLE II

SD OF ERRORS (MSEC) BETWEEN ESTIMATED FPS AND MANUAL ANNOTATIONS FOR DIFFERENT VALUES OF $\epsilon$.

\begin{tabular}{lccccc}
\hline \hline FPs & $\epsilon=1 \%$ & $\epsilon=2 \%$ & $\epsilon=3 \%$ & $\epsilon=4 \%$ & $\epsilon=5 \%$ \\
\hline P on & 22.14 & 21.51 & 20.3 & 19.03 & 18.38 \\
P off & 15.71 & 16 & 16.11 & 16.33 & 16.48 \\
QRS on & 36.98 & 36.23 & 35.17 & 34.09 & 33.36 \\
QRS off & 12.18 & 13.4 & 14.7 & 15.48 & 16.15 \\
T on & 20.02 & 23.64 & 24.21 & 24.49 & 24.39 \\
T off & 19.71 & 18.6 & 19.06 & 18.85 & 19.18 \\
\hline \hline
\end{tabular}

From the values of Tables I and II, we can conclude that as constructed $P 1+P 2$ and $T 1+T 2$ Gaussian functions have almost large amplitude, so by considering a small value for $\epsilon$, we can find onset and offset points for $\mathrm{P}$ and $\mathrm{T}$ waves which satisfied the equations (10) and (11). As we discussed before, for finding the QRSon and QRSoff, we use constructed $Q$ and $S$ Gaussian functions, respectively and as these functions have a small amplitude, for finding the onset and offset points for QRS complex which satisfied (10) and (11), we must consider large value for $\epsilon$. So here, we can not consider unique value for $\epsilon$ for finding onset and offset of all waves : we choose $\epsilon=1 \%$ for $\mathrm{P}$ and $\mathrm{T}$ waves and $\epsilon=5 \%$ for QRS complex.

Lin et al. [2], [3] proposed a Bayesian detection-estimation algorithm for simultaneous detection, delineation, and estimation of $\mathrm{P}$ and $\mathrm{T}$ waves. Their method were based on a Partially Collapsed Gibbs Sampler (PCGS) and they estimate the onset, peak and offset of $\mathrm{P}$ and $\mathrm{T}$ waves and we compare results of our proposed method with their results. For quantitative evaluation of our proposed method ("EKF25new"), we calculate time differences between cardiologist annotations (considered as ground truth) and results of the proposed method for three normal ECG signals of QTDB. Fig. 5 and 6 show the absolute estimation error of "EKF25new" and "PCGS" methods for finding fiducial points of $\mathrm{P}$ and $\mathrm{T}$ waves, respectively. In these figures, beats 1 to 30 are related to "sel17453", beats 31 to 60 are related to "sel16786" and rest of the beats are related to "sel16795". We can see that "EKF25new" can detect FPs with good precision and for some FPs such as $P_{o n}, P_{o f f}$ and $T_{\text {off }}$ the estimation error of "EKF25new" is less than "PCGS" method. Mean (m) and statndard deviation (SD) of estimation errors for these signals are given in tabel III. We can see that for "EKF25new", "m" values for all FPs (except Ton) do not exceed five samples $(20 \mathrm{msec})$ and also for some FPs such as $P_{o n}, P_{o f f}$ and $T_{o f f}$ error of "EKF25new" is very smaller than "PCGS" method.
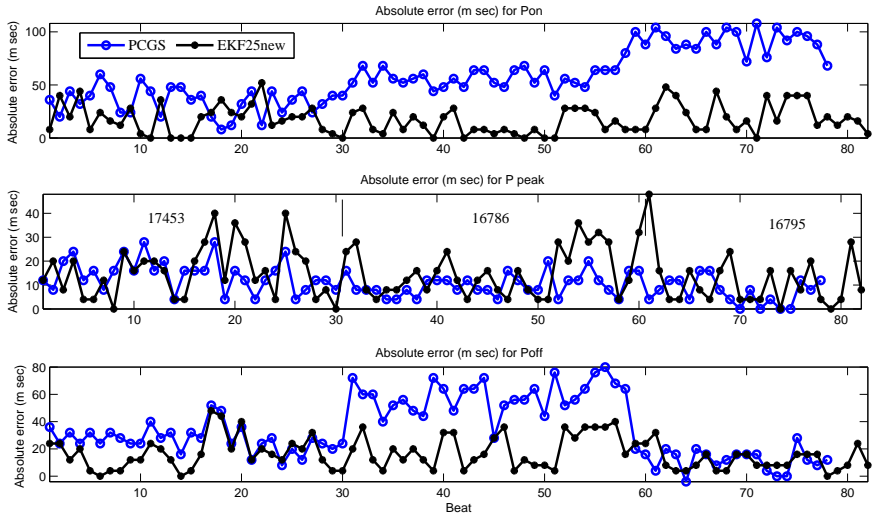

Fig. 5. Absolute Error (msec) for onset, peak and offset of P Wave.

\section{DiscusSiON AND CONCLUSIONS}

In this paper, we proposed a method for extracting fiducial points of ECG signal which is based on a nonlinear dynamic model. By introducing a simple AR model for each of the 21 dynamic parameters of the Gaussian functions and considering separate states for ECG waves, new EKF structure was 

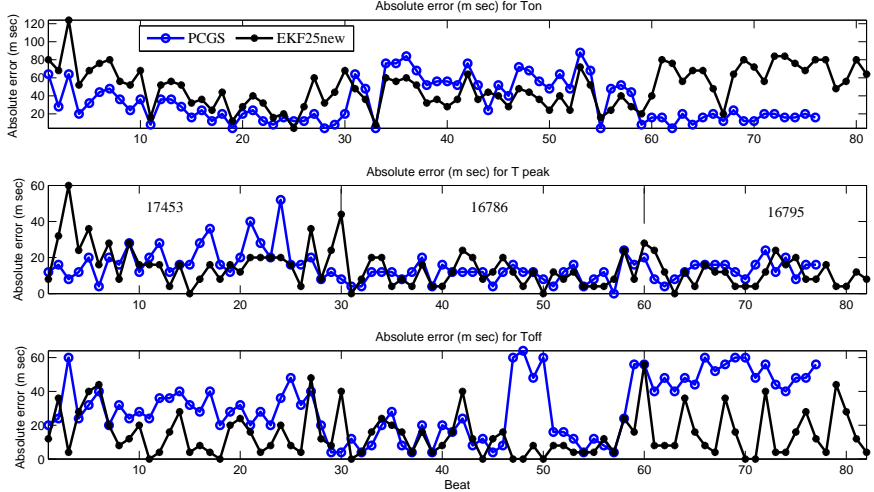

Fig. 6. Absolute Error (msec) for onset, peak and offset of $\mathrm{T}$ Wave.

TABLE III

MEAN AND SD OF ERRORS (MSEC) BETWEEN ESTIMATED FPS AND MANUAL ANNOTATIONS FOR THREE NORMAL ECG SIGNALS.

\begin{tabular}{lcc}
\hline \hline FPs & EKF25new & PCGS \\
\hline P on & $0.97 \pm 22.14$ & $-57.38 \pm 25.33$ \\
P peak & $11.85 \pm 13.8$ & $10.76 \pm 6.9$ \\
P off & $12.73 \pm 15.71$ & $34.72 \pm 21.73$ \\
QRS on & $-17.17 \pm 33.36$ & $N . A$ \\
QRS peak & $-2.25 \pm 5.66$ & $N . A$ \\
QRS off & $12.53 \pm 16.15$ & $N . A$ \\
T on & $47.6 \pm 20.02$ & $31.16 \pm 26.28$ \\
T peak & $9.7 \pm 14.45$ & $14.7 \pm 8.42$ \\
T off & $0.97 \pm 19.71$ & $28.98 \pm 20.63$ \\
\hline \hline
\end{tabular}

constructed. We modify our previous EKF25 model and here we define four observation for the model which this defintion helps the model to estimate the $\mathrm{P}, \mathrm{C}$ and $\mathrm{T}$ waves more correctly and without falling and rising drifts. In this paper we also use the estimated Gaussian parameters to construct a Gaussian function for each wave and use these Gaussian functions for finding the peak, onset and offset of waves. For finding the onset and offset of waves, we benefit the properties of Gaussian functions and by definding a suitable confidence bound $(\epsilon \%)$ for the termination of Gaussian functions, we can detect the onset and offset of waves. Quantitative and qualitative results show that EKF25new approach can detect all the nine FPs (peak, onset and offset of P, QRS and T) and does not miss any one. The mean of estimation error of this method for all FPs (except Ton) do not exceed five samples $(20 \mathrm{msec})$ and in some cases, its results are better than PCGS method. Further work can include the use of this proposed method for analyzing other ECG databases especially abnormal signals and signals which have asymmetrical $\mathrm{P}$ and $\mathrm{T}$ waves.

\section{REFERENCES}

[1] B. Kohler, C. Hennig, and R. Orglmeister, "The principles of software QRS detection: Reviewing and comparing algorithms for detecting this important ECG waveform," IEEE Engineering in Medicine and Biology, pp. 42-57, February 2002, (doi:10.1109/51.993193).

[2] C. Lin, C. Mailhes, and J. Y. Tourneret, "P- and T-wave delineation in ECG signals using a bayesian approach and a partially collapsed gibbs sampler," IEEE Transaction on Biomedical Engineering, vol. 57, no. 12, pp. 2840-2849, December 2010, (doi:10.1109/TBME.2010.2076809).

[3] C. Lin, G. Kail, J. Y. Tourneret, C. Mailhes, and F. Hlawatsch, "P and $\mathrm{T}$ wave delineation and waveform estimation in ECG signals using a block gibbs sampler," in Proc. IEEE Int. Conf. on Acoust., Speech and Sig. Proc. (ICASSP), Prague, Czech Republic, May 2011, pp. 537-540, (doi:10.1109/ICASSP.2011.5946459).

[4] N. P. Hughes, Probabilistic Models for Automated ECG Interval Analysis, Ph.D. thesis, Department of Engineering Science, University of Oxford, 2006.

[5] Y. Sun, K. L. Chan, and Sh. M. Krishnan, "Characteristic wave detection in ECG signal using morphological transform," BMC Cardiovascular Disorders, vol. 5, no. 28, Sept. 2005, (doi:10.1186/1471-2261-5-28).

[6] P. E. McSharry, G. D. Clifford, L. Tarassenko, and L. A. Smith, "A dynamic model for generating synthetic electrocardiogram signals," IEEE Trans. Biomed. Eng., vol. 50, no. 3, pp. 289-294, Mar. 2003, (doi:10.1109/TBME.2003.808805).

[7] R. Sameni, M. B. Shamsollahi, C. Jutten, and G. D. Clifford, "Nonlinear bayesian filtering framework for ECG denoising," IEEE Trans. Biomed. Eng., vol. 54, no. 12, pp. 2172-2185, Dec. 2007, (doi:10.1109/TBME.2007.897817).

[8] O. Sayadi and M. B. Shamsollahi, "A model-based bayesian framework for ECG beat segmentation," Physiol. Meas., vol. 30, pp. 335-352, March 2009, (doi:10.1088/0967-3334/30/3/008).

[9] O. Sayadi, M. B. Shamsollahi, and G. Clifford, "Robust detection of premature ventricular contractions using a wave-based bayesian framework," IEEE Trans. Biomed. Eng., vol. 57, no. 2, pp. 353-362, February 2010, (doi:10.1109/TBME.2009.2031243).

[10] QT Database, http://www.physionet.org/physiobank/database/qtdb/.

[11] P. Laguna, R. G. Mark, A. Goldberg, and G. B. Moody, "A database for evaluation of algorithms for measurement of QT and other waveform intervals in the ECG," IEEE, Computers in Cardiology, vol. 24, pp. 673-676, 1997, (doi:10.1109/CIC.1997.648140).

[12] M. Akhbari, M. B. Shamsollahi, and C. Jutten, "Fiducial points extraction and characteristic waves detection in ECG signal using a model-based bayesian framework," Accepted in 2013 IEEE International Conference on Acoustics, Speech, and Signal Processing (ICASSP2013), May 26 - 31, 2013 ,Vancouver, Canada.

[13] S. M. Kay, Fundamentals of statistical Signal Processing:Estimation Theory, Prentice Hall PTR, 1993, ISBN: 0-13-345711-7.

[14] M. Akhbari, M. B. Shamsollahi, and C. Jutten, "ECG denoising using angular velocity as a state and an observation in an extended kalman filter framework," in 34th Annual International Conference of the IEEE Engineering in Medicine and Biology Society (EMBC2012), San Diego, California, USA, Aug. 2012, pp. 2897 - 2900, (doi:10.1109/EMBC.2012.6346569). 